
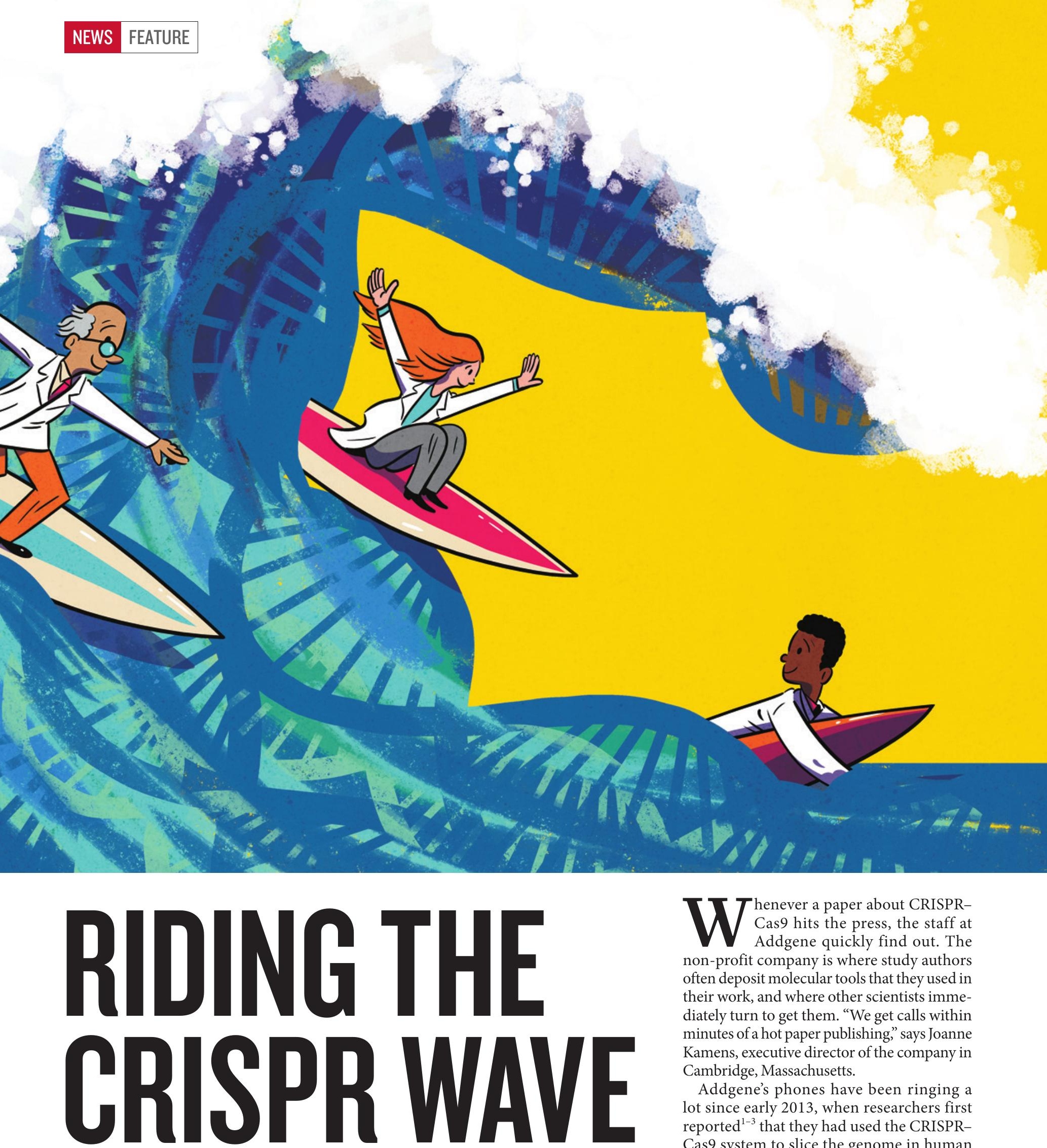

Biologists are embracing the power of

gene-editing tools to explore genomes.

BY HEIDI LEDFORD

W

henever a paper about CRISPRCas9 hits the press, the staff at Addgene quickly find out. The non-profit company is where study authors often deposit molecular tools that they used in their work, and where other scientists immediately turn to get them. "We get calls within minutes of a hot paper publishing," says Joanne Kamens, executive director of the company in Cambridge, Massachusetts.

Addgene's phones have been ringing a lot since early 2013, when researchers first reported $^{1-3}$ that they had used the CRISPRCas9 system to slice the genome in human cells at sites of their choosing. "It was all hands on deck," Kamens says. Since then, molecular biologists have rushed to adopt the technique,

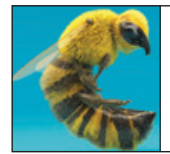

CRISPR EVERYWHERE A Nature special issue nature.com/crispr 


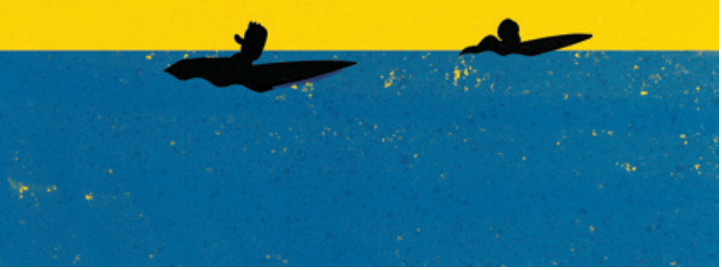

o which can be used to alter the genome of almost any organism with unprecedented ease and finesse. Addgene has sent 60,000 CRISPRrelated molecular tools - about $17 \%$ of its total shipments - to researchers in 83 countries, and the company's CRISPR-related pages were viewed more than one million times in 2015.

Much of the conversation about CRISPRCas9 has revolved around its potential for treating disease or editing the genes of human embryos, but researchers say that the real revolution right now is in the lab. What CRISPR offers, and biologists desire, is specificity: the ability to target and study particular DNA sequences in the vast expanse of a genome. And editing DNA is just one trick that it can be used for. Scientists are hacking the tools so that they can send proteins to precise DNA targets to toggle genes on or off, and even engineer entire biological circuits - with the long-term goal of understanding cellular systems and disease.

"For the humble molecular biologist, it's really an extraordinarily powerful way to understand how the genome works," says Daniel Bauer, a haematologist at the Boston Children's Hospital in Massachusetts. "It's really opened the number of questions you can address," adds Peggy Farnham, a molecular biologist at the University of Southern California, Los Angeles. "It's just so fun."

Here, Nature examines five ways in which CRISPR-Cas9 is changing how biologists can tinker with cells.

\section{BROKEN SCISSORS}

There are two chief ingredients in the CRISPRCas9 system: a Cas9 enzyme that snips through DNA like a pair of molecular scissors, and a small RNA molecule that directs the scissors to a specific sequence of DNA to make the cut. The cell's native DNA repair machinery generally mends the cut - but often makes mistakes.

That alone is a boon to scientists who want to disrupt a gene to learn about what it does. The genetic code is merciless: a minor error introduced during repair can completely alter the sequence of the protein it encodes, or halt its production altogether. As a result, scientists can study what happens to cells or organisms when the protein or gene is hobbled.

But there is also a different repair pathway that sometimes mends the cut according to a DNA template. If researchers provide the template, they can edit the genome with nearly any sequence they desire at nearly any site of their choosing.

In 2012, as laboratories were racing to demonstrate how well these gene-editing tools could cut human DNA, one team decided to take a different approach. "The first thing we did: we broke the scissors," says Jonathan Weissman, a systems biologist at the University of California, San Francisco (UCSF).

Weissman learned about the approach from Stanley Qi, a synthetic biologist now at Stanford University in California, who mutated the Cas9 enzyme so that it still bound DNA at the site that matched its guide RNA, but no longer sliced it. Instead, the enzyme stalled there and blocked other proteins from transcribing that DNA into RNA. The hacked system allowed them to turn a gene off, but without altering the DNA sequence ${ }^{4}$.

The team then took its 'dead' Cas9 and tried something new: the researchers tethered it to part of another protein, one that activates gene expression. With a few other tweaks, they had built a way to turn genes on and off at will ${ }^{5}$.

Several labs have since published variations on this method; many more are racing to harness it for their research ${ }^{6}$ (see 'Hacking CRISPR'). One popular application is to rapidly generate hundreds of different cell lines, each containing a different guide RNA that targets a particular gene. Martin Kampmann, another systems biologist at UCSF, hopes to screen such cells to learn whether flipping certain genes on or off affects the survival of neurons exposed to toxic protein aggregates - a mechanism that is thought to underlie several neurodegenerative conditions, including Alzheimer's disease. Kampmann had been carrying out a similar screen with RNA interference (RNAi), a technique that also silences genes and can process lots of molecules at once, but which has its drawbacks. "RNAi is a shotgun with wellknown off-target effects," he says. "CRISPR is the scalpel that allows you to be more specific."

Weissman and his colleagues, including UCSF systems biologist Wendell Lim, further tweaked the method so that it relied on a longer guide RNA, with motifs that bound to different proteins. This allowed them to activate or inhibit genes at three different sites all in one experiment ${ }^{7}$. Lim thinks that the system can handle up to five operations at once. The limit, he says, may be in how many guide RNAs and proteins can be stuffed into a cell. "Ultimately, it's about payload."

That combinatorial power has drawn Ron Weiss, a synthetic biologist at the Massachusetts Institute of Technology (MIT) in Cambridge, into the CRISPR-Cas9 frenzy. Weiss and his colleagues have also created multiple gene tweaks in a single experiment ${ }^{8}$, making it faster and easier to build complicated biological circuits that could, for example, convert a cell's metabolic machinery into a biofuel factory. "The most important goal of synthetic biology is to be able to program complex behaviour via the creation of these sophisticated circuits," he says.

\section{CRISPR EPIGENETICS}

When geneticist Marianne Rots began her career, she wanted to unearth new medical cures. She studied gene therapy, which targets genes mutated in disease. But after a few years, she decided to change tack. "I reasoned that many more diseases are due to disturbed geneexpression profiles, not so much the single genetic mutations I had been focused on," says Rots, at the University Medical Center Groningen in the Netherlands. The best way to control gene activity, she thought, was to adjust the epigenome, rather than the genome itself.

The epigenome is the constellation of chemical compounds tacked onto DNA and the DNA-packaging proteins called histones. These can govern access to DNA, opening it up or closing it off to the proteins needed for gene expression. The marks change over time: they are added and removed as an organism develops and its environment shifts.

In the past few years, millions of dollars have been poured into cataloguing these epigenetic marks in different human cells, and their patterns have been correlated with everything from brain activity to tumour growth. But without the ability to alter the marks at specific sites, researchers are unable to determine whether they cause biological changes. "The field has met a lot of resistance because we haven't had the kinds of tools that geneticists have had, where they can go in and directly test the function of 


\section{HACKING CRISPR}

By modifying the molecular machinery that powers CRISPR-Cas9 gene editing, scientists can probe the functions of genes and gene regulators with unprecedented specificity.

\section{Snip snip here}

There are two main components of CRISPR-Cas9: the Cas9 enzyme, which cuts DNA, and a snippet of RNA that guides these molecular scissors to the sequence that scientists want to cut

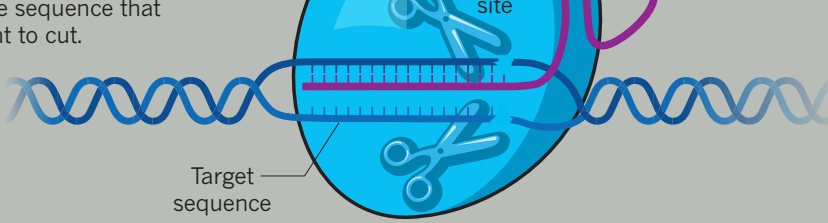

Broken scissors

The Cas9 enzyme can be broken so that it no longer cuts DNA. But with the right guide RNA, it can still attach to specific parts of the genome.

\section{CRISPR inhibition}

A broken, or 'dead', Cas9 enzyme will block the binding of other proteins, such as RNA polymerase, needed to express a gene.

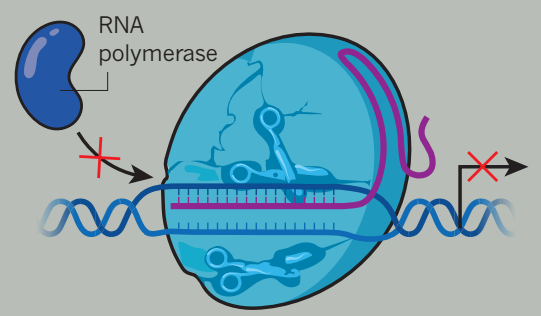

\section{CRISPR activation}

An activating protein can be attached to a dead Cas 9 protein to stimulate expression of a specific gene.

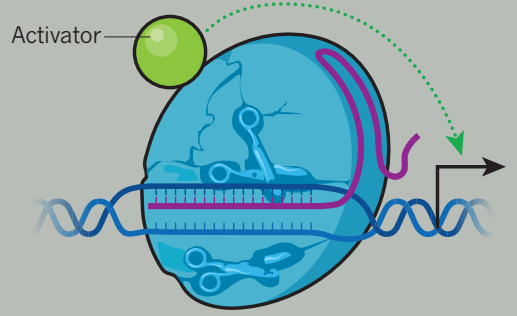

\section{CRISPR epigenetics}

A broken Cas 9 enzyme can be coupled to epigenetic modifiers, such as those that add methy groups (Me) to DNA or acetyl groups (Ac) to histone proteins. This will allow researchers to study how precisely placed modifications affect gene expression and DNA dynamics.
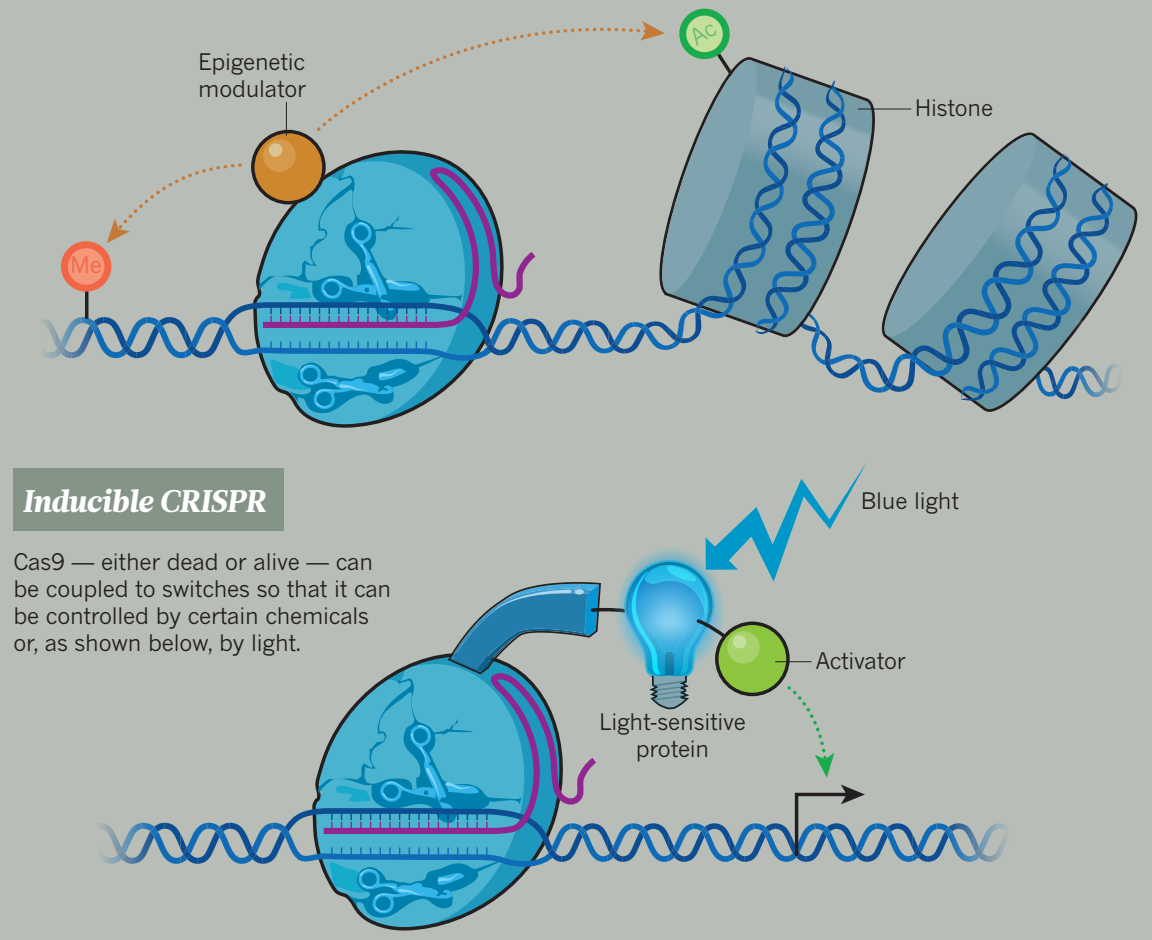

a gene," says Jeremy Day, a neuroscientist at the University of Alabama at Birmingham.

CRISPR-Cas9 could turn things around. In April 2015, Charles Gersbach, a bioengineer at Duke University in Durham, North Carolina, and his colleagues published ${ }^{9}$ a system for adding acetyl groups - one type of epigenetic mark - to histones using the broken scissors to carry enzymes to specific spots in the genome.

The team found that adding acetyl groups to proteins that associate with DNA was enough to send the expression of targeted genes soaring, confirming that the system worked and that, at this location, the epigenetic marks had an effect. When he published the work, Gersbach deposited his enzyme with Addgene so that other research groups could use it - and they quickly did. Gersbach predicts that a wave of upcoming papers will show a synergistic effect when multiple epigenetic markers are manipulated at once.

The tools need to be refined. Dozens of enzymes can create or erase an epigenetic mark on DNA, and not all of them have been amenable to the broken-scissors approach. "It turned out to be harder than a lot of people were expecting," says Gersbach. "You attach a lot of things to a dead Cas9 and they don't happen to work." Sometimes it is difficult to work out whether an unexpected result arose because a method did not work well, or because the epigenetic mark simply doesn't matter in that particular cell or environment.

Rots has explored the function of epigenetic marks on cancer-related genes using older editing tools called zinc-finger proteins, and is now adopting CRISPR-Cas9. The new tools have democratized the field, she says, and that has already had a broad impact. People used to say that the correlations were coincidental, Rots says - that if you rewrite the epigenetics it will have no effect on gene expression. "But now that it's not that difficult to test, a lot of people are joining the field."

\section{CRISPR CODE CRACKING}

Epigenetic marks on DNA are not the only genomic code that is yet to be broken. More than $98 \%$ of the human genome does not code for proteins. But researchers think that a fair chunk of this DNA is doing something important, and they are adopting CRISPR-Cas9 to work out what that is.

Some of it codes for RNA molecules - such as microRNAs and long non-coding RNAs that are thought to have functions apart from making proteins. Other sequences are 'enhancers' that amplify the expression of the genes under their command. Most of the DNA sequences linked to the risk of common diseases lie in regions of the genome that contain non-coding RNA and enhancers. But before CRISPR-Cas9, it was difficult for researchers to work out what those sequences do. "We didn't have a good way to functionally annotate the non-coding genome," says Bauer. "Now our 
experiments are much more sophisticated."

Farnham and her colleagues are using CRISPR-Cas9 to delete enhancer regions that are found to be mutated in genomic studies of prostate and colon cancer. The results have sometimes surprised her. In one unpublished experiment, her team deleted an enhancer that was thought to be important, yet no gene within one million bases of it changed expression. "How we normally classify the strength of a regulatory element is not corresponding with what happens when you delete that element," she says.

More surprises may be in store as researchers harness CRISPR-Cas9 to probe large stretches of regulatory DNA. Groups led by geneticists David Gifford at MIT and Richard Sherwood at the Brigham and Women's Hospital in Boston used the technique to create mutations across a 40,000-letter sequence, and then examined whether each change had an effect on the activity of a nearby gene that made a fluorescent protein $^{10}$. The result was a map of DNA sequences that enhanced gene expression, including several that had not been predicted on the basis of gene regulatory features such as chromatin modifications.

Delving into this dark matter has its challenges, even with CRISPR-Cas9. The Cas9 enzyme will cut where the guide RNA tells it to, but only if a specific but common DNA sequence is present near the cut site. This poses little difficulty for researchers who want to silence a gene, because the key sequences almost always exist somewhere within it. But for those who want to make very specific changes to short, non-coding RNAs, the options can be limited. "We cannot take just any sequence," says Reuven Agami, a researcher at the Netherlands Cancer Institute in Amsterdam.

Researchers are scouring the bacterial kingdom for relatives of the Cas9 enzyme that recognize different sequences. Last year, the lab of Feng Zhang, a bioengineer at the Broad Institute of MIT and Harvard in Cambridge, characterized a family of enzymes called Cpf1 that work similarly to Cas9 and could expand sequence options ${ }^{11}$. But Agami notes that few alternative enzymes found so far work as well as the most popular Cas9. In the future, he hopes to have a whole collection of enzymes that can be targeted to any site in the genome. "We're not there yet," he says.

\section{CRISPR SEES THE LIGHT}

Gersbach's lab is using gene-editing tools as part of an effort to understand cell fate and how to manipulate it: the team hopes one day to grow tissues in a dish for drug screening and cell therapies. But CRISPR-Cas9's effects are permanent, and Gersbach's team needed to turn genes on and off transiently, and in very specific locations in the tissue. "Patterning a blood vessel demands a high degree of control," he says.

Gersbach and his colleagues took their broken, modified scissors - the Cas9 that could now activate genes - and added proteins that are activated by blue light. The resulting system triggers gene expression when cells are exposed to the light, and stops it when the light is flicked off ${ }^{12}$. A group led by chemical biologist Moritoshi Sato of the University of Tokyo rigged a similar system ${ }^{13}$, and also made an active Cas 9 that edited the genome only after it was hit with blue light ${ }^{14}$.

Others have achieved similar ends by combining CRISPR with a chemical switch. the new CRISPR-Cas9 tools to precisely manipulate the genome and epigenome in animal models. "The real power is going to be the integration of those systems," says Dow. This may allow scientists to capture and understand some of the complexity of common human diseases.

Take tumours, which can bear dozens of mutations that potentially contribute to cancer development. "They're probably not all important in terms of modelling a tumour,"

"I wish I had had this technology sooner. My postdoc would have been a lot shorter.'

Lukas Dow, a cancer geneticist at Weill Cornell Medical College in New York City, wanted to mutate cancer-related genes in adult mice, to reproduce mutations that have been identified in human colorectal cancers. His team engineered a CRISPR-Cas9 system in which a dose of the compound doxycycline activates Cas9, allowing it to cut its targets ${ }^{15}$.

The tools are another step towards gaining fine control over genome editing. Gersbach's team has not patterned its blood vessels just yet: for now, the researchers are working on making their light-inducible system more efficient. "It's a first-generation tool," says Gersbach.

\section{MODEL CRISPR}

Cancer researcher Wen Xue spent the first years of his postdoc career making a transgenic mouse that bore a mutation found in some human liver cancers. He slogged away, making the tools necessary for gene targeting, injecting them into embryonic stem cells and then trying to derive mice with the mutation. The cost: a year and US $\$ 20,000$. "It was the rate-limiting step in studying disease genes," he says.

A few years later, just as he was about to embark on another transgenic-mouse experiment, his mentor suggested that he give CRISPR-Cas9 a try. This time, Xue just ordered the tools, injected them into single-celled mouse embryos and, a few weeks later — voilá. "We had the mouse in one month," says Xue. "I wish I had had this technology sooner. My postdoc would have been a lot shorter."

Researchers who study everything from cancer to neurodegeneration are embracing CRISPR-Cas9 to create animal models of the diseases (see page 160). It lets them engineer more animals, in more complex ways, and in a wider range of species. Xue, who now runs his own lab at the University of Massachusetts Medical School in Worcester, is systematically sifting through data from tumour genomes, using CRISPR-Cas9 to model the mutations in cells grown in culture and in animals.

Researchers are hoping to mix and match says Dow. "But it's very clear that you're going to need two or three or four mutations to really model aggressive disease and get closer to modelling human cancer." Introducing all of those mutations into a mouse the old-fashioned way would have been costly and timeconsuming, he adds.

Bioengineer Patrick Hsu started his lab at the Salk Institute for Biological Studies in La Jolla, California, in 2015; he aims to use gene editing to model neurodegenerative conditions such as Alzheimer's disease and Parkinson's disease in cell cultures and marmoset monkeys. That could recapitulate human behaviours and progression of disease more effectively than mouse models, but would have been unthinkably expensive and slow before CRISPR-Cas9.

Even as he designs experiments to genetically engineer his first CRISPR-Cas9 marmosets, Hsu is aware that this approach may be only a stepping stone to the next. "Technologies come and go. You can't get married to one," he says. "You need to always think about what biological problems need to be solved."

Heidi Ledford is a senior reporter for Nature in Cambridge, Massachusetts.

1. Cong, L. et al. Science 339, 819-823 (2013).

2. Mali, P. et al. Science 339, 823-826 (2013).

3. Jinek, M. et al. elife 2, e00471 (2013).

4. Qi, L. S. et al. Cell 152, 1173-1183 (2013).

5. Gilbert, L. A. et al. Cell 159, 647-661 (2014).

6. Dominguez, A. A., Lim, W. A. \& Qi, L. S. Nature Rev. Mol. Cell. Biol. 17, 5-15 (2016)

7. Zalatan, J. G. et al. Cell 160, 339-350 (2015).

8. Kiani, S. et al. Nature Meth. 11, 723-726 (2014).

9. Hilton, I. B. et al. Nature Biotechnol. 33, 510-517 (2015).

10. Rajagopal, N. et al. Nature Biotechnol. 34, 167-174 (2016).

11.Zetsche, B. et al. Cell 163, 759-771 (2015).

12.Polstein, L. R. \& Gersbach, C. A. Nature Chem. Biol. $11,198-200$ (2015).

13. Nihongaki, Y., Yamamoto, S., Kawano, F., Suzuki, H. \& Sato, M. Chem. Biol. 22, 169-174 (2015).

14.Nihongaki, Y., Kawano, F., Nakajima, T. \& Sato, M. Nature Biotechnol. 33, 755-760 (2015).

15.Dow, L. E. et al. Nature Biotechnol. 33, 390-394 (2015). 